\title{
Comment on Cheng et al.: Intramedullary nailing via suprapatellar approach versus locked plating of proximal extra-articular tibial fractures: a randomized control trial
}

\author{
Hao Yin ${ }^{1}$ (D) \\ Received: 1 October 2020 / Accepted: 16 October 2020 / Published online: 20 October 2020 \\ (C) SICOT aisbl 2020
}

Dear Editor,

We read with great interest the recent article entitled "Intramedullary nailing via suprapatellar approach versus locked plating of proximal extra-articular tibial fractures: a randomized control trial" published online in 26 September 2020 issue of International Orthopaedics by Cheng et al [1].The authors compared two treatment methods in terms of their effectiveness and safety. They considered that both IMN through the suprapatellar approach and minimally invasive LCP were found to yield no significant intergroup difference of clinical outcomes in the treatment of proximal, extra-articular tibial fractures. It is a valuable study. Nevertheless, we have several suggestions and queries that we would like to communicate with the authors.

1 Regarding the timing of surgery, emergency surgery is a good choice before severe limb swelling, especially for closed fractures, and emergency surgery within four or 12 hours can obviously reduce complications $[2,3]$. However, the author did not mention the timing of surgery in the paper. If there is a difference in the timing of surgery between the two groups, it will have a certain impact on the effect of surgery and complications.

2 Questions about soft tissue conditions. Just as mentioned by the authors, this type of fracture is usually caused by highenergy injury, and soft tissue injury is more serious. As we all know, severe fractures can lead to tension blisters around the fracture site, and these tension blisters can affect the healing of the incision even if they are absorbed and the scab is formed. The authors did not specify the soft tissue conditions when the surgery is performed. It is generally believed that the suprapatellar approach for intramedullary nail can be made far away from the fracture site, and the soft tissue

Hao Yin

helloyh2008@126.com

1 Department of Orthopaedics, The Third Affiliated Hospital of An Hui Medical University, Hefei, China conditions around the fracture have little influence on it, which is also one of its advantages. However, MIPO method has strict requirements on soft tissue conditions. Even if subperiosteal dissection and LCP is placed under the injured soft tissue, it is easy to cause poor wound healing or even infection. Nevertheless, the author's clinical results showed no difference in the infection rate between the two groups, which was quite puzzling. We hope the author could give a better explanation.

3 There is a slip of the pen in this paper. In Table 1, the total number of OTA classification in IMN Group is $154(41+$ $53+60)$ instead of sample size 152. In the LCP Group, the total number of OTA classification is $159(39+49+71)$ instead of sample size 154 . In the mechanism of Injury column, the total number of LCP Group is $159(69+74$ +16 ) instead of sample size 154. It was just a simple error. We hope the authors can check it carefully in the course of the reviewing process.

In conclusion, the results of this paper by Cheng et al. [1] should be interpreted with caution. To reach a definitive conclusion, further high-quality RCTs based on larger sample sizes are still required.

\section{References}

1. Cheng L, Li Y, Chu Y et al (2020) Intramedullary nailing via suprapatellar approach versus locked plating of proximal extraarticular tibial fractures: a randomized control trial. Int Orthop. https://doi.org/10.1007/s00264-020-04821-5

2. Xu YQ, Li Q, Shen TG et al (2013) An efficacy analysis of surgical timing and procedures for high-energy complex tibial plateau fractures. Orthop Surg 5(3): 188-195

3. Tang X, Liu L, Tu CQ et al (2012) Timing of internal fixation and effect on Schatzker IV-VI tibial plateau fractures. Chin J Traumatol 15(2):81-85

Publisher's note Springer Nature remains neutral with regard to jurisdictional claims in published maps and institutional affiliations. 